\title{
A VISITA PRÉ-OPERATÓRIA DE ENFERMAGEM PELA ENFERMEIRA DO CENTRO CIRÚRGICO *
}

SLIVA, A. A visita pré-operatória de enfermagem pela enfermeira do centro cirúrgico. Rev. Esc. Enf. USP, São Paulo, 21(2):145-160, ago. 1987.

Este trabalho teve por finalidade verificar o número de hospitais com enfermeiras de centro cirúrgico, em São Paulo, que realizam a visita pré-operatória de enfermagem, procedimento este indispensável para assegurar ao paciente assistência continua e centrada em suas necessidades. Os resultados mostraram que a maioria das enfermeiras, embora tendo conhecimento da importância desse procedimento, não o realizam.

UNITERMOS: Cuidado pré-operatório. Enfermagem em centro-cirúrgico. Cuidados de enfermagem.

\section{Histórico}

Toda pessoa, ao ser admitida num hospital, traz consigo muitas dúvidas e preocupações em relação ao seu futuro e à recuperação de sua saúde.

Quando o tratamento cirúrgico se faz necessário as reações psicológicas do paciente são exacerbadas, pois este terá de enfrentar o desconhecido.

KAMTYAMA 18 declara que "todo ser humano tem medo do desconhecido: o que é desconhecido gera medo e insegurança, por ser indefinivel, imprevisível e incontrolável".

O paciente cirúrgico tem medo da cirurgia, de sentir dor, da anestesia, de nảo acordar da anestesia, da solidão, dos aparelhos e equipamentos, do resultado da operação e da morte (ALCOFORADO et alii ${ }^{1}$; FONTES et alii ${ }^{12}$; GONÇALVES ${ }^{13}$; LOTTERMANN ${ }^{21}$; MENEZES ${ }^{25}$; PLEITEZ ${ }^{27}$; SANTOS \& CABERLON ${ }^{30}$ ).

Suas inquietações e ansiedades vão aumentando progressivamente à medida que se aproxima a data da cirurgia.

JOUCLAS ${ }^{16}$, citando vários autores, refere que "as horas e minutos que antecedem a cirurgia podem ser de agonia para o paciente, levan-

* Trabalho orientado pela Dra. Sonia Della Torre Salzano, Professor Titular do Departa. mento de Enfermagem Médico-Cirúrgica da Escola de Enfermagem da USP.

** Enfermeira. Auxiliar de Ensino do Departamento de Enfermagem Médico-Cirúrgica da Escola de Enfermagem da USP - disciplina Enfermagem em Centro-Cirúrgico. 
do-o até o pânico, o que poderá resultar em sérios problemas pós-operatórios".

ALCOFORADO et alii ${ }^{1}$ acrescentam ainda, que, enquanto o paciente está aguardando o momento da cirurgia, sua angústia e estresse emocional podem aumentar e acarretar crises hipertensivas que poderão retardar ou mesmo suspender a cirurgia programada.

Segundo Luckmann citado por GONÇALVES ${ }^{13}$, os pacientes muito ansiosos no período pré-operatório têm maior possibilidade de um pósoperatório mais problemático do que aqueles que se apresentam relativamente calmos.

Uma explicação sobre todos os procedimentos nos quais o paciente estará envolvido seria de grande auxílio para diminuir o medo, a insegurança e apreensão por ele sentidos. (PLEITZ ${ }^{27}$, SCHMITT \& WOOLDRIDGE ${ }^{31}$ ).

McPHAIL ${ }^{23}$ acredita ser a enfermeira a profissional mais indicada para assistir integralmente ao paciente, identificando suas necessidades básicas e planejando os cuidados para atender a estas necessidades, enquanto RODRIGUES ${ }^{28}$ enfatiza a importância do papel da enfermeira na equipe cirúrgica, para assegurar ao paciente assistência individualizada e integral.

Vários autores concordam que, para a identificação das necessidades básicas e o planejamento de cuidados individualizados, a enfermeira precisa conhecer o paciente e vencer a barreira física e psicológica que existe separando a unidade de internação e a do centro cirủrgico (ALEXANDER et alii ${ }^{2}$; ATKINSON ${ }^{3}$; CASTELLANOS ${ }^{5}$; CLEMONS ${ }^{7}$; FERRAZ ${ }^{9}$; HARTSON \& HARTSON ${ }^{14}$; JOUCLAS ${ }^{16}$; MEHAFFY ${ }^{24}$; MENEZES ${ }^{25}$; PANZA ${ }^{26}$; SALZANO ${ }^{29}$; WALLIS ${ }^{35}$ ).

A necessidade da visita da enfermeira do centro cirúrgico ao paciente que restá aguardando o momento da cirurgia tem sido defendida por vários autores. Dessa forma, ela poderá colher informaçôes e identificar as necessidades do paciente para planejar e implementar os cuidados de enfermagem individualizados nos períodos pré e transoperatório, e assim prover a continuidade de assistência de enfermagem ,ALEXANDER et alii ${ }^{2}$; ATKINSON ${ }^{3}$; BIANCHI \& CASTELLANOS ${ }^{4}$; CASTELLANOS \& BIANCHI ${ }^{6}$; FEHLAU 8; FIELD ${ }^{11}$; HOOPES \& MCCONNELL 15; LINDEMAN 19; LINDEMAN \& STETZER 20; MAHOMET ${ }^{22}$; PANZA ${ }^{26}$; SALZANO ${ }^{29}$; SANTOS \& CABERLON ${ }^{30}$; SCHRADER ${ }^{32}$; SHETLER ${ }^{33}$; THOMSON ${ }^{34}$; WALLIS ${ }^{35}$ ).

BIANCHI \& CASTELLANOS ${ }^{4}$, tecendo considerações sobre a visita pré-operatória de enfermagem, afirmam que esse "é o momento de interação enfermeira-paciente, com o intuito de prestar a melhor assistência de enfermagem a que o paciente tem direito", e, citando inúmeros autores, determinam os objetivos da visita pré-operatória de enfermagem: dignidade:

- "respeitar a individualidade de paciente, proteger seus direitos e 
- promover a continuidade do cuidado de enfermagem entre a unidade de internação e o centro cirúrgico;

- estabelecer um vínculo de comunicação entre a unidade de internação e o centro cirúrgico;

levantar dados para promover, recuperar e manter o estado de saúde do indivíduo;

- estabelecer o diagnóstico de enfermagem, formular objetivos e planejar os cuidados para o periodo transoperatório;

- verificar a orientação dada pelo cirurgião e anestesista, esclarecendo rotinas e procedimentos relacionados à proposta anestésica-cirúrgica;

- promover a interação da enfermeira de centro cirúrgico com o paciente, procurando conhecer sua ansiedade e apreensões, e expectativas de cuidado;

- reforçar informações recebidas da equipe de saude;

- incrementar a segurança do paciente pelo conhecimento de mais um membro da equipe cirúrgica;

- aumentar o grau de satisfação da enfermeira de Centro Cinúrgico no seu trabalho, em virtude de maior contato direto com o paciente".

PANZA 26 acredita ser a visita pré-operatória de enfermagem, realizada pela enfermeira do centro cirúrgico, "um procedimento único para uma assistência de enfermagem efetiva, pois a enfermeira torna-se o elemento coordenador da assistência de enfermagem. Através do entrosamento com o paciente, ela levanta e avalia suas necessidades; com a enfermeira da unidade, "checa" as necessidades e cuidados pré-operatórios; com o anestesista comunica os receios e ansiedades do paciente; com o cirurgião, verifica e prevê material adequado para o procedimento cirúrgico e, finalmente, com a equipe de enfermagem, distribui e coordena as atividades na sala de cirurgia".

O ideal é que a enfermeira de centro cirúrgico realize a visita pré-operatória de enfermagem, para detectar as necessidades do paciente e proporcionar-lhe assistência individualizada e efetiva.

Certos autores fazem algumas restrições para a realização da visita pré-operatória de enfermagem.

ATKINSON ${ }^{3}$ relata certa resistência por parte das enfermeiras das unidades de internação, que questionam a necessidade da enfermeira do centro cirúrgico precisar conhecer o paciente.

De acordo com CLEMONS ${ }^{1}$, algumas pessoas são de opinião que, com a visita pré-operatória, a enfermeira de centro cirúrgico pode interferir na área de atuação do cirurgião e do anestesista. 
ALEXANDER et alii ${ }^{2}$ apontam que algumas enfermeiras estão relutantes em deixar o centro cirúrgico, onde se sentem seguras, e evitam contato com o paciente. Acrescentam, ainda, que algumas estão interessadas em fazer a visita, mas não sabem como elaborar um programa ou vencer a resistência que possam encontrar dos médicos ou mesmo das enfermeiras da unidade de internação.

SALZANO ${ }^{29}$ relata que a visita pré-operatória de enfermagem não está sendo realizada pela enfermeira do centro cirúrgico devido ao "pequeno número de enfermeiras que atuam nessa unidade e pela sobrecarga técnico-administrativa que se impõe à enfermeira como parte de sua responsabilidade".

Segundo PANZA ${ }^{26}$, algumas vezes a enfermeira recebe o paciente no centro cirúrgico sem ter tido contato anterior com ele, sem ter informações e dados quanto ao seu estado emocional, suas dúvidas, o que dificulta o planejamento adequado de cuidados no trans e pós operatório.

F'ERRAZ 10, em seu trabalho sobre as "expectativas e opiniões do paciente cirúrgico quanto ao cuidado de enfermagem no período transo-peratório", demonstrou que a maioria dos pacientes relatam não terem visto a enfermeira do centro cirúrgico no período transoperatório. Isto mostra que a enfermeira dessa unidade "está delegando funções e nem os pacientes a conhecem".

RODRIGUES 28 demonstra, em seu estudo, que a enfermeira do centro cirúrgico está muito distante do paciente e raramente a visita pré-operatória. Relata, também, que o paciente cirúrgico sente a falta de alguém que lhe esclareça as dúvidas, lhe explique como é a sala de operações e converse com ele para diminuir seu temor e angústia.

JOUCLAS \& SALZANO ${ }^{17}$ propõem a utilização de uma ficha que contenha, de forma sistematizada, as informações necessárias para o planejamento do cuidado de enfermagem ao paciente no periodo transoperatório, porque a enfermeira do Centro Cirúrgico não tem conhecimento do paciente e de suas necessidades.

Sugerem, também, que o ideal seria a utilização dessa ficha como um recurso para facilitar a visita pré-operatória de enfermagem.

A enfermeira de centro cirúrgico deve assegurar, ao paciente, assistência de enfermagem centrada nas necessidades deste, e uma das formas de conseguí-lo é por meio da visita pré-operatória.

Por sentirmos que este procedimento não tem merecido a devida atenção por parte das enfermeiras que atuam na unidade de centro cirúrgico, e preocupadas com esta situação, principalmente em relação ao conhecimento das mesmas formal ou não, da conveniência da visita pré-operatória de enfermagem, para proporcionar ao paciente cirúrgico assistência contínua, individualizada e eficaz, é que nos propusemos realizar o presente estudo. 


\section{2 - OBJETIVOS}

Este trabalho tem como objetivos:

1 - Verificar se as enfermeiras de centro cirúrgico tiveram alguma experiência educacional relativa à visita pré-operatória de enfermagem;

2 - Verificar quantas enfermeiras de centro cirúrgico realizam a visita pré-operatória de enfermagem;

3 - Identificar o número de hospitais em que as enfermeiras de centro cirúrgico nealizam a visita pré-operatória de enfermagem.

\section{3 - METODOLOGIA}

\section{População}

A população deste estudo foi constituída por 30 enfermeiras que estavam atuando na unidade de centro cirúrgico, em 14 hospitais do Distrito de São Paulo, no período de novembro de 1983 a fevereiro de 1984 .

\section{Tamanho da amostra}

Por se tratar de um estudo de caráter exploratório, os parâmetros tradicionalmeite utilizados para a delimitação do tamanho da amostra perdem a sua relevância.

Assim, consideramos que uma amostra constituida por cerca de $20 \%$ dos elementos da população (hospitais) seria suficiente, em termos de representatividade, para os fins destes estudo.

O processo de amostragem utilizado foi o sistemático, após prévia ordenação das unidades amostrais.

\section{Seleção da amostra}

A amostra foi selecionada a partir de uma listagem de todos os hospitais do Distrito de São Paulo, fornecida pelo Serviço de Registro e Cadastro do Departamento de Técnica Hospitalar:

Dos 47 hospitais escolhidos em duas seleções, 14 constituíram a população deste estudo, por contarem com centro cirúrgico na qual havia enfermeiras; foram excluídos 17 que não tinham centro cirúrgico, 15 que tinham centro cirúrgico mas sem enfermeiras nessa área, e 1 que se encontrava co mo centro cirúrgíco desativado, em reforma.

Os 14 hospitais contavam com 30 enfermeiras no centro cirúrgico assim distribuidas: 10 hospitais com uma enfermeira, 1 com duas, 1 com três, 1 com cinco e 1 com dez enfermeiras. 
Para a coleta de dados foi utilizado um questionário (Anexo 1) com perguntas abertas e fechadas, composto de duas partes.

$\mathrm{Na}$ parte I, foram incluídos os dados relativos aos cursos realizados de enfermagem e, também, o tempo de serviço na área de centro cirúrgico; e, na parte II, perguntas relativas à experiência educacional sobre a visita pré-operatória de enfermagem, e se a enfermeira de centro cirúrgico estava realizando este procedimento.

\section{Coleta de dados}

Para a coleta de dados, foi mantido contato telefônico com as Chefes dos Serviços de Enfermagem dos hospitais selecionados, com a finalidade de determinar o dia e a hora de entrega do questionário, para que as enfermeiras de aentro cirúrgico pudessem preenchê-lo.

$\mathrm{Na}$ maioria dos hospitais, o questionário foi preenchido e devolvido prontamente mas, em alguns, houve necessidade de deixá-lo para ser preenchido e devolvido posteriormente, pelo fato das enfermeiras trabalharem em horários diferentes.

Análise dos dados.

Os dados obtidos foram analisados com base em indices percentuais.

\section{4 - RESULTADOS E COMENTARIOS}

Os resultados serão apresentados e comentados na seguinte ordem:

I - Características da população.

II - Experiência educacional sobre a visita pré-operatória de enfermagem.

III - A realização da visita pré-operatória de enfermagem pela enfermeira do centro cirúrgico.

\section{I - Características da população}

Das 30 enfermeiras da população em estudo, $8(26,6 \%)$ têm menos de 2 anos de formada, $7(23,3 \%)$ têm de 6 a 8 anos, e $5(16,7 \%)$ de 8 a 10 anos. A maioria das enfermeiras, $26(86,7 \%)$, concluiu Curso de Graduação há menos de 10 ảnos.

Em relação à de Habilitação, verificamos que $20(66,6 \%)$ enfermeiras fizeram este curso e $10(33,3 \%)$, não.

As áreas de Habilitação cursadas pelas enfermeiras foram: Enfermagem Médico-Cirúrgica, 11; Licenciatura em Enfermagem, 10; Enfermagem em Saúde Pública, 4 e Enfermagem Obstétrica, duas.

Observamos que 7 fiezram duas habilitações, sendo a segunda sempre na área de Licenciatura em Enfermagem. 
TABEL A 1

NUMERO E PORCENTAGEM DE ENFERMEIRAS, SEGUNDO OS CURSOS DE ATUALIZAÇAO, DE APERFETÇOAMENTO E DE ESPECIALIZAÇAO, QUE FIZERAM. SAO PAULO, 1984.

\begin{tabular}{|c|c|c|c|c|c|c|}
\hline \multirow{3}{*}{ Enfermeiras } & \multicolumn{6}{|c|}{ Cursos } \\
\hline & \multicolumn{2}{|c|}{ Aturalização } & \multicolumn{2}{|c|}{ Aperfeiçoamento } & \multicolumn{2}{|c|}{ Especializacãac } \\
\hline & $\mathbf{N}^{\mathbf{9}}$ & $\%$ & $\mathbf{N}^{\mathrm{P}}$ & $\%$ & $\mathbf{N o}$ & $\%$ \\
\hline Sim & 1 & 3,3 & 1 & 3,3 & 14 & 46,7 \\
\hline Não & 29 & 96,7 & 29 & 96,7 & 16 & 53,3 \\
\hline Total & 30 & 100,0 & 30 & 100,0 & 30 & 100,0 \\
\hline
\end{tabular}

Podemos verificar que apenas uma enfermeira $(3,39)$ fez Curso de Atualização "Enfermagem em Cardiologia" e uma, (3,3\%), de Aperfeiçoamento "Enfermagem em Unidade de Terapia Intensiva", enquanto que $14(46,7)$ enfermeiras fiezram Curso de Especialização, sendo a área mais procurada a de Administração Hospitalar, 11. As outras áreas cursadas foram Enfermagem do Trabalho, Enfermagem Obstétrica e Enfermagem em Recuperação Pós-Anestésica.

Nenhuma enfermeira fez Curso de Pós-Graduação, "sensu stricto".

TABLA 2

NUMERO E PORCENTAGEM DE ENFERMEIRAS, SEGUNDO O TEMPO DE ATUAÇAO NA UNIDADE DE CENTTRO CIRÚRGICO. S.AO PAULO, 1984.

\begin{tabular}{ccc}
\hline & \multicolumn{2}{c}{ Enfermeiras } \\
\hline $\begin{array}{c}\text { Tempo de Atuação } \\
\text { (anos) }\end{array}$ & No & $\%$ \\
\hline $0-\mid 2$ & 16 & 53,3 \\
$2-\mid 4$ & 4 & 13,3 \\
$4-\mid 6$ & 4 & 13,3 \\
$6-\mid 8$ & 3 & 10,0 \\
$8-\mid 10$ & 2 & 6,7 \\
em branco & 1 & 3,3 \\
\hline Tot a l & 30 & 99,9 \\
\hline
\end{tabular}

Pelos dados desta tabela observamos que $16(53,3 \%)$ enfermeiras têm menos de 2 anos de atuação no centro cirúrgico, e que todas as enfermeiras têm menos de 10 anos de atuação nessa unidade.

Pelos resultados obtidos verificamos que, da população estudada; $86,7 \%$ das enfermeiras têm menos de 10 anos de formada; $66,6 \%$ fizeram o Curso de Habilitação, sendo a área de maior freqüência a de Enfer- 
magem Médico-Cirúrgica; $3,3 \%$ têm Curso de Atualização e de Aperfeiçoamento; $46,7 \%$ cursaram Especialização, 11 das quais o fizeram na área de Administração Hospitalar.

Da população estudada $53,3 \%$ têm menos de 2 anos de atuação no centro cirúrgico.

Das 30 enfermeiras pertencentes ao estudo, nenhuma tem o Curso de Pós-Graduação "Sensu sttricto".

II - Experiência educacional sobre visita pré-operatória de enfermagem, das 30 enfermeiras que fazem parte deste estudo

26 enfermeiras $(86,6 \%$ têm conhecimento da visita préoperatória de enfermagem. Destas $19(63,3 \%)$ objetiveram tal conhecimento durante os Cursos de Graduação sendo 14 no Curso Geral de Graduação, uma Habilitação, e 4 em ambos: 7 enfermeiras $(23,3 \%)$ tiveram dela conhecimento, 7 somente através de outras fontes: bibliografia, reuniões científicas, encontros, palestras, e $4(13,3 \%)$ não têm qualquer conhecimento deste procedimento.

T A B E L A 3

NUMERO E PORCENTAGEM DE ENFERMEIRAS, SEGUNDO O TEMPO DE FORMADA E O CONHECIMENTO SOBRE A VISITA PRE-OPERATORIA DE ENFERMAGEM, OBTTDO DURANTE O CURSO DE ENFERMLAGEM OU SOMENTE ATRAVES DE OUTRAS FONTES. SAO PAULO, 1984.

\begin{tabular}{|c|c|c|c|c|c|c|c|c|}
\hline \multirow{4}{*}{$\begin{array}{c}\text { Tempo de formada } \\
\text { (anos) }\end{array}$} & \multicolumn{8}{|c|}{$\begin{array}{l}\text { Conhecimento } \\
\text { Fonte de conhecimento } \\
\text { Enfermeiras }\end{array}$} \\
\hline & \multicolumn{4}{|c|}{ Sim } & \multicolumn{2}{|c|}{ Não } & \multicolumn{2}{|c|}{ Total } \\
\hline & \multicolumn{2}{|c|}{$\begin{array}{c}\text { Cursos de } \\
\text { Enfermagem }\end{array}$} & \multicolumn{2}{|c|}{$\begin{array}{l}\text { Outras } \\
\text { Fontes }\end{array}$} & \multirow[b]{2}{*}{$\mathbf{N}^{e}$} & \multirow[b]{2}{*}{$\%$} & \multirow[b]{2}{*}{$N^{9}$} & \multirow[b]{2}{*}{$\%$} \\
\hline & $N^{9}$ & $\%$ & $N^{9}$ & $\%$ & & & & \\
\hline $0-12$ & 6 & 20,0 & 1 & 3,3 & 1 & 3,3 & 8 & 26,6 \\
\hline $2-4$ & 3 & 10,0 & - & - & - & - & 3 & 10,0 \\
\hline $4-j 6$ & 2 & 6,7 & 1 & 3,3 & - & - & 3 & 10,0 \\
\hline $6-8$ & 5 & 16,6 & 2 & 6,7 & - & - & 7 & 23,3 \\
\hline $8-10$ & 3 & 10,0 & 2 & 6,7 & - & - & 5 & 16,7 \\
\hline 10 ou mais & - & - & 1 & 3,3 & 3 & 10,0 & 4 & 13,3 \\
\hline Total & 19 & 63,3 & 7 & 23,3 & 4 & 13,3 & 30 & 99,9 \\
\hline
\end{tabular}

Pelos dados desta tabela, podemos verificar que das enfermeiras que conheciam a visita pré-operatória de enfermagem como procedimento a ser realizado pela enfermeira de centro cirúrgico, $7(23,3 \%)$ têm de $0-\mid 2$ anos de formada e 7 ) $23,3 \%$ ), têm de $6 \longrightarrow \mid 8$ anos. 
As $19(63,3 \%)$ enfermeiras que obtiveram este conhecimento durante o Curso de Enfermagem, têm menos de 10 anos de formada.

Observamos, ainda, que dentre as $4(13,3 \%)$ enfermeiras que não tiveram conhecimento sobre a visita pré-operatória de enfermagem, quer durante os Cursos de Enfermagem quer através de outras fontes, 3 (10,0\%) concluíram o Curso de Graduação há mais de 10 anos.

Isto nos permite inferir que, nos últimos anos, a abordagem do ensino de Enfermagem em Centro Cirúrgico tem dado maior enfoque sobre a atuação da enfermeira de centro cirúrgico, na área expressiva.

O que mais nos chama a atenção é o fato de $4(13,3 \%)$ enfermeiras não conhecerem este procedimento através de outras fontes, tais como revistas, livros, reunióes científicas e outras, uma vez que, nos últimos anos, este assunto tem sido mais divulgado tanto na literatura estrangeira como na nacional, e em encontros científicos.

III - Realização da visita pré-operatória de enfermagem pela enfermeira do centro cirúrgico

Das $30(100,0 \%)$ enfermeiras do estudo, apenas $3(10 \%)$ enfermeiras, uma em cada hospital, fazem a visita pré-operatória de enfermagem, e 27 (90\%) não realizam este procedimento.

Nos 3 hospitais em que este procedimento é realizado, um conta com 10 enfermeiras atuando na unidade de centro cirúrgico, outro com duas enfermeiras e o terceiro com apenas uma enfermeira.

TABELA 4

NOMERO E PORCENTAGEM DE ENFERMEIRAS, SEGUNDO O TEMPO DE FORMADA E A REALIZAÇAO DA VISITA PRE-OPERATORIA DE ENFERMAGEM. SAO PAULO, 1984.

Enformoiras

Realizacáo

\begin{tabular}{|c|c|c|c|c|c|c|}
\hline \multirow{2}{*}{$\begin{array}{c}\text { Tempo de formada } \\
\text { (anos) }\end{array}$} & \multicolumn{2}{|c|}{ Sim } & \multicolumn{2}{|c|}{ Não } & \multicolumn{2}{|c|}{ Total } \\
\hline & $N$ & $\%$ & $\mathbf{N}^{\circ}$ & $\%$ & Ne & $\%$ \\
\hline $0 \longrightarrow 2$ & 3 & 10,0 & $5)$ & 16,7 & 8 & 26,7 \\
\hline $2 \longrightarrow 4$ & - & - & 3 & 10,0 & 3 & 10,0 \\
\hline $4-16$ & - & - & 3 & 10,0 & 3 & 10,0 \\
\hline $6-18$ & - & - & 7 & 23,3 & 7 & 23,3 \\
\hline $8 \longrightarrow 10$ & - & - & $5]$ & 16,7 & 5 & 16,7 \\
\hline 10 ou mais & - & - & 4 & 13,3 & 4 & 13,3 \\
\hline Total & 3 & 10,0 & 27 & 90,0 & 30 & 100,0 \\
\hline
\end{tabular}


Os dados desta tabela mostram que as $3(10,0 \%)$ enfermeiras que fazem a visita pré-operatória de enfermagem, concluíram o Curso de Graduação há menos de 2 anos, e, dentre as $27(90,0 \%)$ que não a realizam, $23(76,7 \%)$ concluíram o Curso de Graduação há menos de 10 anos.

Pelos resultados obtidos, observamos que apenas uma pequena parcela da população, $3(10,0 \%)$ enfermeiras deste estudo têm realizado a visita pré-operatória de enfermagem que, acreditamos, seja um procedimento muito importante para proporcionar ao cliente melhor qualidade de assistência.

Isto vem corroborar as afirmações de SALZANO ${ }^{29}$ e SANTOS \& CABERLON ${ }^{30}$, quando afirmam que este procedimento é pouco adotado em nosso País, e dão como prováveis justificativas, o número reduzido de profissionais que atuam na área de centro cirúrgico, a sobrecarga administrativa e gerencial que se impõe à enfermeira dessa unidade, e, ainda, o fato das enfermeiras não terem incorporado a importância deste procedimento, da metodologia e dos resultados que são obtidos quando a visita pré-operatória de enfermagem é realizada.

Verificamos, também, que não é somente a falta de conhecimento sobre este procedimento que faz com que as enfermeiras de centro cirúr. gico não realizem a visita pré-operatória de enfermagem, pois a grande maioria, $26(86,6 \%)$, afirmou ter conhecimento desta atividade, embora apenas $3(10,0 \%)$ enfermeiras a realizem.

Segundo a opinião das enfermeiras que fazem parte deste estudo, a visita pré-operatória de enfermagem é um procedimento muito importante a ser realizado pela enfermeira de centro cirúrgico, pois ajuda a diminuir o estresse e a ansiedade do paciente, podendo levá-lo à maior tranquilidade e confiança, por este saber que encontrará alguém conhecido nessa unidade. Acrescentam, ainda, que este procedimento fornece subsídios para o planejamento e implementação de assistência individualizada ao paciente no período transoperatório; facilita a previsão do material necessário para a realização do ato anestésico-cirúrgico; propicia efetiva interação enfermeira-paciente e melhor relacionćmento entre as enfermeiras do centro cirúrgico e as das unidades de internação.

As dificuldades mencionadas por essas enfermeiras como causa para a não realização da visita pré-operatória de enfermagem são: pouca disponibilidade de tempo, principalmente quando o número de cirurgias é grande e existe apenas uma enfermeira atuando no centro cirúrgico; responsabilidade por outras unidades, além do centro cirúrgico; paciente cuja internação ocorre no dia da cirurgia, o que dificulta a realização da visita pré-operatória de enfermagem, pois ela é solicitada no centro cirúrgico durante a realização da programação cirúrgica.

Como podemos perceber, as enfermeiras estão conscientes da importância deste procedimento, mas as barreiras encontradas para a operacionalização do mesmo fazem com que raramente realizem esta atividade 
que poderia lhes trazer a maior satisfação no trabalho, assim como melho. ria na qualidade de assistência para o paciente cirúrgico.

Acreditamos que essas barreiras mencionadas pelas enfermeiras só serão gradativamente superadas a partir do momento em que elas incorporarem efetivamente o valor da visita pré-operatória de enfermagem como procedimento essencial para a prestação de assistência de enfermagem, individualizada e eficaz ao paciente cirúrgico.

\section{CONCLUSŐES}

Este trabalho nos permite concluir que:

- $19(63,3 \%)$ enfermeiras tiveram conhecimento a respeito da visita pré-operatória de enfermagem durante os Cursos de Enferma. gem, sendo que:

- $14(46,6 \%)$ enfermeiras conheceram este procedimento durante o Curso Geral de Graduação;

- uma $(3,3)$, durante o Curso de Habilitação;

$4(13,3 \%)$, no Curso de Graduação e na Habilitação;

- $7(23,3 \%)$ adquiriram este conhecimento através de outras fontes, como bibliografia, palestras e reuniões cientificas;

- $4(13,3 \%)$ não tiveram conhecimento a respeito da visita pré-operatória de enfermagem;

- $3(10,0 \%)$ enfermeiras de centro cirúrgico realizam a visita pré-operatória de enfermagem e $27(90,0 \%)$ não;

- Em $3(21,4 \%)$ hospitais, a visita pré-operatória de enfermagem é realizada pelas enfermeiras de centro cirúrgico, e em 11 $(78,6 \%)$, este procedimento não tem sido realizado.

SILVA, A. The pre-operative nursing assessment of patients by the operating room nurses. Rev. Esc. Enf. USP, São Paulo, 21(2):145-160, Aug. 1987.

The objective of this survey is to verify how many São Paulo hospitals which employ operating room nurses, proceed in having pre-operative nursing assessment of patients. This procedure is essencial to assure a continuous nursing assistance and basic to patient-needs centered nursing care. Results found show that the majority of nurses although knowing about the importance of the procedure, do nof implement it.

UNITERMS: Pre-operative care. Operating room nursing. Nursing care.

\section{REFERENCIAS BIBLIOGRAFICAS}

1. ALCOFORADO, L.M.A. et alil. Humanização no atendimento do paciente cirúrgico. Enfoque, São Paulo, 5 (n̊ especial): 7-9, jun. 1976.

2. ALEXANDER, C. et alii. Preoperative visits: the OR nurse unmasks. AORN J., Denver, 19(2):401-12, Feb. 1974.

3. ATKInson, L.J. The circle of patient care. AORN J., Denver, 16(3):45-50, Sept. 1972. 
4. BIANCHI, E.R.F. \& CASTELLANOS, B.E.P. Considerações sobre a visita pré-operatória do enfermeiro da unidade de centro cirúrgico: resenha da literatura estrangeira. Rev. paul. Enf., São Paulo, 3(5):161-6, out./dez. 1983.

5. CASTellanos, B.E.P. Aplicação do processo de enfermagem ao cuidado do paciente na unidade de centro cirúrgico. Rev. Esc. Enf. USP., São Paulo, 12(3):170-86, dez. 1978.

6. CASTEllanos, B.E.P. \& BIANCHI, E.R.F. Visita pré-operatória do enfermeiro da unidade de centro cirúrgico: marcos referenciais para o seu ensino no curso de graduacão de enfermagem. Rev. paul. Enf., São Paulo, 4(1):10-4, jan/mar. 1984.

7. CLEMONS, B. The OR nurs in the patient care circuit. Am. J. Nurs., New York, 68(10): 2141-4, Oct. 1968.

8. FEHLAU, M.T. Applying the nursing process to patient care in the operating room. Nurs. Clin. North Am., Philadelphia, 10(4):617-23, Dec. 1975.

9. FERRaz, E.R. Focalizando o paciente no centro cirúrgico. Rev. Esc. Enf. USP., São Paulo, 12(3):167-9, dez. 1978.

10.

- O paciente cirúrgico: suas expectativas e opiniões quanto ao cuidado de enfermagem no período transoperatório. Rev. Bras. Enf., Brasilia, 35(1):48-59, jan./mar. 1982.

11. FIELD, L.W. Identifying the psychological aspects of the surgical patient. AORN J., Denver, 17(1):86-90, Jan. 1973.

12. FONTES, M. de C.C. et alii. O trauma cirúrgico: importância da orientação pré-operatória. Rev. Bras. Enf., Brasília, 33(2):194-200, abr./jun. 1980.

13. GONÇALVES, M.M.C. Enfermagem e segurança emocional do paciente. Enf. Novas Dimens., São Paulo, 5(1):34, jan. 1979.

14. HARTSON, D. \& HARTSON, K.M. The five-minute interview. AORN J., Denver, 31(4): 605-8, Mar. 1980.

15. HOOPES, N.M. \& MCCONNELL, M. An approach to preoperative visits. AORN J., Denver, 26(6):1048-52, Dec. 1977.

16. JOUCLAS, V.M.G. Elaboração e avaliação de um instrumento de comunicação que favoreça a assistência de enfermagem no transoperatório. São Paulo, 1977. 85p. (Dissertação de mestrado - Escola de Enfermagem da USP).

17. JOUClas, V.M.G. \& SALZANO, S.D.T. Planejamento de uma ficha pré-operatória de enfermagem. Rev. Esc. Enf. USP., São Paulo, 15(1) :5-16, abr. 1981.

18. KAMIYAMA, $Y$, O doente hospitalizado e sua percepcão quanto à prioridade de seus problemas. São Paulo, 1972. 111p. (Tese de doutorado - Escola de Enfermagem da USP)

19. LINDEMAN, C.A. Study evaluates effects of preoperative visits. AORN J., Denver, $19(2): 427-37$, Feb. 1974 .

20. LINDEMAN, C.A. \& STETZER, S.L. Effect of preoperative visits by operating room nurses. Nurs. Res., New York, 22(1):4-16, Jan./Feb. 1973.

21. LotTERMANN, c. Enfermagem e segurança emocional. Rev. Gaúcha Enf. Porto Alegre, 3(2):127-32, jun. 1982.

22. MAHOMET, A.D. Nursing diagnosis for the OR nurse. AORN J., Denver, 22(5):709-11, Nov. 1975.

23. McPHAIL, J.L. A plea for the professional nurse in the OR. AORN J., Denver, 19(4): 872-6, Apr. 1974. 
24. MEHAFFY, N.L. Assessment and communication for continuity of care for the surgical patient. Nurs. Clin. North Am., Philadelphia, 10(4):625-33, Dec. 1975.

25. MENEZES, A.R. de. A problemática de enfermagem dos pacientes no periodo transoperatório: um estudo dos problemas sentidos e observados. São Paulo, 1978. 81 p. (Dissertação de mestrado - Escola de Enfermagem da USP).

26. PANZA, A.M.M. Efeito da visita pré-operatória da enfermeira de centro cirúrgico sobre o estresse do paciente no pré-operatório, no dia da cirurgia e no pós-operatório. São Paulo, 1977. 75p. (Dissertação de mestrado - Escola de Enfermagem da USP).

27. PLEITEZ, J.A. Psychoolgical complications of the surgical patient. AORN J., Denver, 16(2): 137-46, Aug. 1972.

28. RODRIGUES, A.I. O paciente no sistema centro cirúrgico: um estudo sobre as percepções e opiniões dos pacientes em relação ao período transoperatório. São Puulo, 1979. 150 p. (Dissertação de mestrado - Escola de Enfermagem da USP).

29. SALZANO, S.D.T. Instrumento de comunicação de enfermagem. Estudo da implantação de um modelo de comunicação escrita entre as equipes de enfermagem das unidades cirúrgicas e do centro cirúrgico. São Paulo, 1982. 102 p. (Tese de Livre Docência Escola de Enfermagem da USP).

30. SANTOS, E. da S. \& CABERLON, I.C. Visita pré e pós-operatória aos pacientes. Enfoque, São Paulo, 9(6):41-5, dez. 1981.

31. SCHMITT, F.E. \& WOOLDRIDGE, P.J. Psychological preparation of surgical patients. Nurs. Res., New York, 22(2):108-15, Mar./Apr. 1973.

32. SCHRADER, S.E. Is the preop visit a nursing function? AORN J., Denvèr, 19(2):375-6, Feb. 1974.

33. SHETLER, M.G. Oprating room nurses go visiting. Am. J. Nurs., New York, 72(7):1266-9, July 1972.

34. THOMSON, E. Preop visits - for the nurse - for the patient? AORN J., Denver, 16(4): :75-81, Oct. 1972.

35. WALLIS, R.M. Preoperative visits: a challenge for OR nurses. AORN J., Denver, 14(6) :53-6, Dec. 1971.

Recebido para publicação em 24-11-86

Aprovado para publicação em 18-08-87 
Anexo 1

\section{QUESTIONARIO}

\section{PARTE I}

1 - Dados educacionais.

1.1. Cursos de enfermagem.

1.1.1. Graduação

\section{A) Geral:}

- Nome da Escola:

- Ano do início do curso:

- Ano do término do curso:

B) Habilitação:

- Area:

- Nome da Escola:

- Ano do início do curso:

- Ano do término do curso:

1.1.2. Atualização (mínimo de $40 \mathrm{hs.-aula)}$

- Area:

- Nome da Escola:

- Ano do término:

1.1.3. Pós-Graduação ("Sensu lato")

A) Aperfeiçoamento (mínimo de 180 hs.-aula)

- Area:

- Nome da Escola:

- Ano do término:

B) Especialização (minimo de 360 hs.-aula)

- Area:

- Nome da Escola:

- Ano do término:

1.1.4. Pós-Graduação ("Sensu stricto")
A) Mestrado
- Area:
- Nome da Escola:
- Ano do término: 


\section{B) Doutorado}

- Area:

- Nome da Escola:

- Ano do término:

1.2. Outros cursos (Nivel Superior)

- Nome do Curso:

- Nome da Escola:

- Ano do término:

- Nome do Curso:

- Nome da Escola:

- Ano do término:

2 - Dados profissionais.

2.1. Tempo de serviço no centro cirúrgico.

Anos

Meses

Dias.

\section{PARTE II}

1 - Durante o(s) seu(s) Curso(s) de Enfermagem, foi-lhe oferecida alguma experiência educacional relativa à visita pré-operatória de enfermagem, que deve ser realizada pelo(a) enfermeiro(a) do centro cirúrgico?
A) $\operatorname{sim}$
B) Não

Nota: Se a resposta foi NAO passe para a pergunta $n^{\circ} 4$.

2 - Se sua resposta foi $S I M$, indique em que curso(s).

\subsection{Graduação}
A) Geral:
B) Habilitação:

2.2. Atualização:

2.3. Pós-Graduação ("Sensu lato")

A) Aperfeiçoamento:

B) Especialização:

2.4. Pós-Graduação ("Sensu stricto")
A) Mestrado:
B) Doutorado:

3 - Além do(s) Curso(s) de Enfermagem, existiram outras fontes que enriqueceram o seu conhecimento sobre a visita pré-operatória de enfermagem?
A) $\mathbf{S i m}$
B) Não

Se a resposta foi SIM, indique quais foram essas fontes: 
4 - Se a resposta da pergunta $n^{\circ} 1$ fol NAO, diga se voce tem conhecimento sobre a visita pré-operatória de enfermagem.
A) Sim
B) Não

Se a resposta foi SLM, indique como adquiriu esse conhecimento:

5 - Na instituição em que trabalha é realizada a visita pré-operatória de enfermagem pelo(a) enfermeiro(a) do centro cirúrgico?
A) $\operatorname{Sim}$
B) Não

Nota: Se a resposta foi NAO, passe para a pergunta $n^{\circ} 8$.

6 - A visita pré-operatória de enfermagem é realizada a todos os pacientes que irão se submeter a cirurgia programada?
A) $\operatorname{Sim}$
B) Não

Justifique sua resposta:

7 - Você, como enfermeiro(a) deste centro cirúrgico, realiza a visita pré-operatória de enfermagem?
A) $\operatorname{Sim}$
B) Não

Justifique sua resposta:

8 - Qual a sua opinião sobre a visita pré-operatória de enfermagem como procedimento que deve ser realizado pelo(a) enfermeiro(a) do centro cirúrgico? 